\title{
PERTURBATION OF EIGENVALUES OF MATRIX PENCILS AND OPTIMAL ASSIGNMENT PROBLEM
}

\author{
MARIANNE AKIAN, RAVINDRA BAPAT, AND STÉPHANE GAUBERT
}

\begin{abstract}
We consider a matrix pencil whose coefficients depend on a positive parameter $\epsilon$, and have asymptotic equivalents of the form $a \epsilon^{A}$ when $\epsilon$ goes to zero, where the leading coefficient $a$ is complex, and the leading exponent $A$ is real. We show that the asymptotic equivalent of every eigenvalue of the pencil can be determined generically from the asymptotic equivalents of the coefficients of the pencil. The generic leading exponents of the eigenvalues are the "eigenvalues" of a min-plus matrix pencil. The leading coefficients of the eigenvalues are the eigenvalues of auxiliary matrix pencils, constructed from certain optimal assignment problems.
\end{abstract}

Perturbation de valeurs propres de faisceaux matriciels et problème d'affectation optimale

\begin{abstract}
RÉsumÉ. Nous considérons un faisceau matriciel dont les coefficients, dépendant d'un paramètre $\epsilon$, ont des équivalents asymptotiques de la forme $a \epsilon^{A}$, lorsque $\epsilon$ tend vers zéro par valeurs positives, le coefficient dominant $a$ étant complexe et l'exposant dominant $A$ étant réel. Nous montrons qu'un équivalent asymptotique pour chacune des valeurs propres du faisceau peut être déterminé génériquement à partir des équivalents des coefficients du faisceau. Les exposants dominants des valeurs propres sont les valeurs propres d'un faisceau matriciel min-plus, et les coefficients dominants sont les valeurs propres de faisceaux auxiliaires, construits au moyen de problèmes d'affectation optimale.
\end{abstract}

\begin{abstract}
Abridged French version
Nous considérons un faisceau matriciel $\mathcal{A}_{\epsilon}=\mathcal{A}_{\epsilon, 0}+X \mathcal{A}_{\epsilon, 1}+\cdots+X^{d} \mathcal{A}_{\epsilon, d}$, où $X$ est une indéterminée, et où pour tout $0 \leq k \leq d, \mathcal{A}_{\epsilon, k}$ est une matrice $n \times$ $n$ dont les coefficients, $\left(\mathcal{A}_{\epsilon, k}\right)_{i j}$, sont des fonctions continues à valeurs complexes d'un paramètre positif $\epsilon$. On s'intéresse au comportement asymptotique, lorsque $\epsilon$ tend vers 0 , des valeurs propres $\mathcal{L}_{\epsilon}$ de $\mathcal{A}_{\epsilon}$, qui sont par définition les racines du polynôme $\operatorname{det}\left(\mathcal{A}_{\epsilon}\right)$. Nous supposons que pour tout $0 \leq k \leq d$, on a des matrices $a_{k}=\left(\left(a_{k}\right)_{i j}\right) \in \mathbb{C}^{n \times n}$ et $A_{k}=\left(\left(A_{k}\right)_{i j}\right) \in(\mathbb{R} \cup\{+\infty\})^{n \times n}$ telles que $\left(\mathcal{A}_{\epsilon, k}\right)_{i j}=$ $\left(a_{k}\right)_{i j} \epsilon^{\left(A_{k}\right)_{i j}}+o\left(\epsilon^{\left(A_{k}\right)_{i j}}\right)$, pour $1 \leq i, j \leq n$. Lorsque $\left(A_{k}\right)_{i j}=+\infty$, cela signifie, par convention, que $\left(\mathcal{A}_{\epsilon, k}\right)_{i j}$ est nulle au voisinage de 0 . Nous cherchons un équivalent

Date: February 23, 2004.

2000 Mathematics Subject Classification. Primary 47A55. Secondary 47A75, 15A22, 05C50, $12 \mathrm{~K} 10$.

Key words and phrases. Perturbation theory, max-plus algebra, tropical semiring, spectral theory, Newton-Puiseux theorem, amoeba, graphs, optimal assignement problem, Hungarian algorithm, WKB asymptotics, large deviations. Mots clés. Théorie des perturbations, algèbre max-plus, semi-anneau tropical, théorie spectrale, théorème de Newton-Puiseux, amibe, graphes, problème d'affectation optimale, algorithme Hongrois, asymptotiques WKB, grandes déviations.
\end{abstract}


asymptotique de la forme $\mathcal{L}_{\epsilon} \sim \lambda \epsilon^{\Lambda}$, avec $\lambda \in \mathbb{C} \backslash\{0\}$ et $\Lambda \in \mathbb{R}$, pour chaque valeur propre $\mathcal{L}_{\epsilon}$ de $\mathcal{A}_{\epsilon}$.

Lorsque $\mathcal{A}_{\epsilon}=\mathcal{A}_{\epsilon, 0}-X$ id, où id désigne la matrice identité, il s'agit du problème classique de perturbation de valeur propres de matrices. En particulier, lorsque la matrice $\mathcal{A}_{\epsilon, 0}$ est de la forme $\mathcal{A}_{\epsilon, 0}=\mathcal{A}_{0,0}+\epsilon b$, pour une matrice complexe $b=\left(b_{i j}\right)$, une théorie initiée par Višik et Ljusternik VL60, et complétée par Lidskiı̌ [Lid65] identifie les exposants dominants $\Lambda$, pour des valeurs génériques des paramètres $b_{i j}$, et donne des formules explicites pour les coefficients dominants $\lambda$, faisant intervenir les valeurs propres de certains compléments de Schur construits à partir de $b$. Voir [MBO97 pour une présentation récente. Cependant, pour certaines valeurs de $b$, ces compléments de Schur peuvent ne pas exister. De tels cas singuliers ont été étudiés en particulier dans MBO97, ME98. Dans ABG04, nous avons donné une première extension du théorème de Lidskiǔ Lid65, donnant sous certaines conditions structurelles les équivalents des valeurs propres de la matrice $\mathcal{A}_{\epsilon, 0}$. Nous étendons ici ce résultat au cas des faisceaux, ce qui permet de résoudre les cas qui demeuraient singuliers dans [ABG04.

Afin d'énoncer le résultat principal, rappelons quelques notions d'algèbre minplus. Le semi-anneau min-plus, $\mathbb{R}_{\text {min }}$, est l'ensemble $\mathbb{R} \cup\{+\infty\}$ muni de l'addition $(a, b) \mapsto a \oplus b:=\min (a, b)$ et de la multiplication $(a, b) \mapsto a \otimes b:=a+b$. On écrira parfois $a b$ au lieu de $a \otimes b$. On notera $\mathbb{O}:=+\infty$ et $\mathbb{1}:=0$ le zéro et l'unité de $\mathbb{R}_{\min }$, respectivement.

Nous associons au faisceau $\mathcal{A}_{\epsilon}$ le faisceau matriciel $A=A_{0} \oplus X A_{1} \oplus \cdots \oplus X^{d} A_{d}$ à coefficients dans $\mathbb{R}_{\text {min }}$. Les coefficients de $A, A_{i j}$, sont donc des polynômes formels à coefficients dans $\mathbb{R}_{\min }$, en l'indéterminée $X$. Nous appelons polynôme caractéristique min-plus le permanent $P_{A}=\operatorname{perm} A$. Rappelons que pour une matrice $B=\left(B_{i j}\right)$ de taille $n \times n$, à coefficients dans un semi-anneau quelconque, le permanent de $B$ est défini comme la somme sur toutes les permutations $\sigma$ du poids $|\sigma|_{B}=B_{1 \sigma(1)} \cdots B_{n \sigma(n)}$. Lorsque les coefficients de $B$ appartiennent à $\mathbb{R}_{\min }$, $|\sigma|_{B}=B_{1 \sigma(1)}+\cdots+B_{n \sigma(n)}$, et perm $B$ est la valeur d'une affectation optimale dans le graphe valué associé à $B$.

Si $P$ est un polynôme formel à coefficients dans $\mathbb{R}_{\min }$, on note $\hat{P}$ la fonction polynôme associée à $P$. L'application $P \mapsto \hat{P}$ est un cas particulier de transformée de Legendre-Fenchel BCOQ92 § 3.3.1]. Cuninghame-Green et Meijer CGM80 ont montré que la fonction polynôme $\hat{P}(x)$ peut se factoriser de manière unique sous la forme $\hat{P}(x)=a\left(x \oplus c_{1}\right) \cdots\left(x \oplus c_{n}\right)$, avec $a, c_{1}, \ldots, c_{n} \in \mathbb{R}_{\text {min }}$. Les nombres $c_{1}, \ldots, c_{n}$ sont appelés racines de $P$. Nous noterons $\gamma_{1}, \ldots, \gamma_{N}$ les racines du polynôme caractéristique $P_{A}$, aussi appelées valeurs propres du faisceau $A$. Les racines $\gamma_{i}$, et donc la fonction polynôme $\hat{P}_{A}$, peuvent être calculés en temps $O\left(n^{4} d\right)$ en s'inspirant de la méthode de Burkard et Butkovič [BB03.

Pour toute matrice $B \in \mathbb{R}_{\min }^{n \times n}$ telle que perm $B \neq \mathbb{0}$, nous définissons le graphe $\operatorname{Opt}(B)$ formé des arcs participant à une affectation optimale : les nœuds de $\operatorname{Opt}(B)$ sont $1, \ldots, n$, et il y a un arc de $i$ à $j$ s'il y a une permutation $\sigma$ telle que $j=\sigma(i)$ et $|\sigma|_{B}=\operatorname{perm} B$.

Nous dirons que deux vecteurs $U, V$ de dimension $n$ à coefficients dans $\mathbb{R}_{\min } \backslash\{\mathbb{O}\}$ forment une paire hongroise relativement à $B$ si, quels que soient $i, j$, on a $B_{i j} \geq$ $U_{i} V_{j}$, et si $U_{1} \cdots U_{n} V_{1} \cdots V_{n}=\operatorname{perm} B$, les produits s'entendant dans le semianneau min-plus. Ainsi, $(U, V)$ n'est autre qu'une solution optimale du problème linéaire dual du problème d'affectation (see [BR97, 2.4]). En particulier, une paire 
hongroise existe dès que perm $B \neq \mathbb{0}$, et elle peut être trouvée en temps $O\left(n^{3}\right)$ grâce à l'algorithme hongrois. Pour toute paire hongroise $(U, V)$, on définit le graphe de saturation, $\operatorname{Sat}(B, U, V)$, qui a pour nœuds $1, \ldots, n$, et pour $\operatorname{arcs}$ les couples $(i, j)$ tels que $B_{i j}=U_{i} V_{j}$.

Pour chaque racine finie $\gamma$ de $P_{A}$, nous définissons les graphes $\operatorname{Opt}_{0}(\gamma), \ldots, \operatorname{Opt}_{d}(\gamma): \operatorname{Opt}_{k}(\gamma)$ a pour nœuds $1, \ldots, n$, et a un arc de $i$ à $j$ si $(i, j) \in \operatorname{Opt}(\hat{A}(\gamma))$ et $\left(A_{k}\right)_{i j} \gamma^{k}=\hat{A}_{i j}(\gamma)$. Pour toute paire hongroise $(U, V)$ relativement à la matrice $\hat{A}(\gamma)$, nous définissons aussi les graphes $\operatorname{Sat}_{0}(\gamma, U, V), \ldots, \operatorname{Sat}_{d}(\gamma, U, V)$, qui sont obtenus en $\operatorname{remplaçant} \operatorname{Opt}(\hat{A}(\gamma))$ par $\operatorname{Sat}(\hat{A}(\gamma), U, V)$ dans la définition $\operatorname{de} \operatorname{Opt}_{0}(\gamma), \ldots, \mathrm{Opt}_{d}(\gamma)$. Enfin, si $G$ est un graphe ayant pour nœuds $1, \ldots, n$, et si $b \in \mathbb{C}^{n \times n}$, nous définissons la matrice $b^{G}$, telle que $\left(b^{G}\right)_{i j}=b_{i j}$ si $(i, j) \in G$, et $\left(b^{G}\right)_{i j}=0$ sinon. Dans l'énoncé du théorème qui suit, les valeurs propres sont comptées avec leurs multiplicités.

Théorème 1. Soit $\gamma$ une racine finie du polynôme caractéristique min-plus $P_{A}$. Pour chaque $0 \leq k \leq d$, notons $G^{k}$ le graphe égal a $\operatorname{Opt}_{k}(\gamma)$ ou bien à $\operatorname{Sat}_{k}(\gamma, U, V)$, pour un choix quelconque de la paire hongroise $U, V$ relativement à $\hat{A}(\gamma)$. Considérons le faisceau auxiliaire $a^{(\gamma)}:=a_{0}^{G_{0}}+X a_{1}^{G_{1}}+\cdots+X^{d} a_{d}^{G_{d}}$. Alors, si le faisceau $a^{(\gamma)}$ a $m_{\gamma}$ valeurs propres non-nulles, $\lambda_{1}, \ldots, \lambda_{m_{\gamma}}$, le faisceau $\mathcal{A}_{\epsilon}$ admet $m_{\gamma}$ valeurs propres $\mathcal{L}_{\epsilon, 1}, \ldots, \mathcal{L}_{\epsilon, m_{\gamma}}$ ayant des équivalents respectifs de la forme $\mathcal{L}_{\epsilon, i} \sim \lambda_{i} \epsilon^{\gamma}$. En outre, si 0 est une valeur propre de multiplicité $m_{\gamma}^{\prime}$ du faisceau $a^{(\gamma)}$, le faisceau $\mathcal{A}_{\epsilon}$ admet $m_{\gamma}^{\prime}$ valeurs propres supplémentaires $\mathcal{L}_{\epsilon}$ telles que $\epsilon^{-\gamma} \mathcal{L}_{\epsilon}$ converge vers 0 , et toutes les autres valeurs propres $\mathcal{L}_{\epsilon}$ de $\mathcal{A}_{\epsilon}$ sont telles que le module de $\epsilon^{-\gamma} \mathcal{L}_{\epsilon}$ tend vers l'infini. Enfin, pour des valeurs génériques des paramètres $\left(a_{k}\right)_{i j}, m_{\gamma}$ coïncide avec la multiplicité de la racine $\gamma$, et $m_{\gamma}^{\prime}$ coïncide avec la somme des multiplicités des racines de $P_{A}$ strictement supérieures à $\gamma$.

Dans la version en anglais de la présente note, nous illustrons le Théorème 1en raffinant des résultats de Najman Naj99.

We consider a matrix pencil of the form

$$
\mathcal{A}_{\epsilon}=\mathcal{A}_{\epsilon, 0}+X \mathcal{A}_{\epsilon, 1}+\cdots+X^{d} \mathcal{A}_{\epsilon, d},
$$

where, for every $0 \leq k \leq d, \mathcal{A}_{\epsilon, k}$ is a $n \times n$ matrix whose coefficients, $\left(\mathcal{A}_{\epsilon, k}\right)_{i j}$, are complex valued continuous functions of a nonnegative parameter $\epsilon$, and $X$ is an indeterminate. We are interested in the asymptotic behavior, when $\epsilon$ tends to 0 , of the eigenvalues $\mathcal{L}_{\epsilon}$ of $\mathcal{A}_{\epsilon}$, which are the roots of the polynomial $\operatorname{det}\left(\mathcal{A}_{\epsilon}\right)$.

We shall assume that for every $0 \leq k \leq d$, matrices $a_{k}=\left(\left(a_{k}\right)_{i j}\right) \in \mathbb{C}^{n \times n}$ and $A_{k}=\left(\left(A_{k}\right)_{i j}\right) \in(\mathbb{R} \cup\{+\infty\})^{n \times n}$ are given, so that

$$
\left(\mathcal{A}_{\epsilon, k}\right)_{i j}=\left(a_{k}\right)_{i j} \epsilon^{\left(A_{k}\right)_{i j}}+o\left(\epsilon^{\left(A_{k}\right)_{i j}}\right), \quad \text { for all } 1 \leq i, j \leq n,
$$

when $\epsilon$ tends to 0 . When $\left(A_{k}\right)_{i j}=+\infty$, this means by convention that $\left(\mathcal{A}_{\epsilon, k}\right)_{i j}$ is zero in a neighborhood of zero. We look for an asymptotic equivalent of the form $\mathcal{L}_{\epsilon} \sim \lambda \epsilon^{\Lambda}$, with $\lambda \in \mathbb{C} \backslash\{0\}$ and $\Lambda \in \mathbb{R}$, for every eigenvalue $\mathcal{L}_{\epsilon}$ of $\mathcal{A}_{\epsilon}$.

When $\mathcal{A}_{\epsilon}=\mathcal{A}_{\epsilon, 0}-X$ id, where id denotes the identity matrix, we recover the classical problem of perturbation of eigenvalues of matrices. In particular, when $\mathcal{A}_{\epsilon, 0}$ is affine in $\epsilon$, i.e. $\mathcal{A}_{\epsilon, 0}=\mathcal{A}_{0,0}+\epsilon b$, for some complex matrix $b=\left(b_{i j}\right)$, a theory initiated by Višik and Ljusternik VL60 and completed by Lidskiu Lid65 identifies 
the leading exponents $\Lambda$ for generic values of the parameters $b_{i j}$, and gives explicit formulæ for the leading coefficients $\lambda$, involving the eigenvalues of certain Schur complements constructed from $b$. See [MBO97 for a recent presentation. For some values of $b$, however, these Schur complements may not be defined. Such singular cases have received much attention, see in particular MBO97, [ME98. In [ABG04, we generalized the theorem of Lid65] to the case where $\mathcal{A}_{\epsilon, 0}$ is not affine in $\epsilon$. We showed that the leading exponents $\Lambda$ of the eigenvalues can be computed, generically, as the "roots" of a certain min-plus polynomial constructed from $\mathcal{A}_{\epsilon}$. We also showed that under structural conditions (when certain bipartite graphs constructed from the leading exponents of the entries of $\mathcal{A}_{\epsilon, 0}$ have a perfect matching), the leading exponents of all the eigenvalues can be obtained by Schur complement formulæ which extend the ones of Lid65]. However, when these structural conditions do not hold, the question remained open. We show in Theorem 1 below that the leading coefficients of the eigenvalues of the pencil $\mathcal{A}_{\epsilon}$ are obtained, generically, as the eigenvalues of certain auxiliary pencils (independent of $\epsilon$ ), constructed by solving certain optimal assignment problems. This gives a complete generalization of the theorem of Lid65. We shall illustrate Theorem 1 in Corollary 1 by refining results of Najman Naj99 concerning a singular perturbation of an affine pencil.

In order to state the main result, we need some min-plus algebraic constructions. Recall that the min-plus semiring, $\mathbb{R}_{\min }$, is the set $\mathbb{R} \cup\{+\infty\}$ equipped with the addition $(a, b) \mapsto \min (a, b)$ and the multiplication $(a, b) \mapsto a+b$. We denote by " $\oplus$ " the min-plus addition, and by " $\otimes$ " or concatenation the min-plus multiplication. We denote by $\mathbb{O}=+\infty$ and $\mathbb{1}=0$ the zero and unit elements of $\mathbb{R}_{\min }$, respectively. The max-plus semiring, $\mathbb{R}_{\max }$, is obtained by replacing "min" by "max" and $+\infty$ by $-\infty$ in this definition.

We associate to the matrix pencil $\mathcal{A}_{\epsilon}$ the matrix pencil with coefficients in $\mathbb{R}_{\min }$, $A=A_{0} \oplus X A_{1} \oplus \cdots \oplus X^{d} A_{d}$. Here, the entries of $A, A_{i j}$, are formal polynomials in the indeterminate $X$ with coefficients in $\mathbb{R}_{\min }$. We consider the formal min-plus characteristic polynomial $P_{A}=\operatorname{perm} A$. Recall that for a $n \times n$ matrix $B=\left(B_{i j}\right)$ with entries in any semiring, the permanent of $B$ is defined as the sum over all permutations $\sigma$ of the weight $|\sigma|_{B}=B_{1 \sigma(1)} \cdots B_{n \sigma(n)}$. Thus, if $B$ is a matrix with entries in $\mathbb{R}_{\min }$, the weight of $\sigma,|\sigma|_{B}$, is the usual sum $B_{1 \sigma(1)}+\cdots+B_{n \sigma(n)}$, and $\operatorname{perm} B$ is the value of an optimal assignment in the weighted graph associated to $B$. (See BR97] for more background.)

If $P$ is a formal polynomial with coefficients in $\mathbb{R}_{\min }$, we denote by $\hat{P}$ the polynomial function associated to $P$. The map $P \mapsto \hat{P}$ is a specialization of the LegendreFenchel transform BCOQ92, § 3.3.1]. Cuninghame-Green and Meijer CGM80. have shown that the min-plus polynomial function $\hat{P}(x)$, can be factored uniquely as $\hat{P}(x)=a\left(x \oplus c_{1}\right) \cdots\left(x \oplus c_{n}\right)$. with $a, c_{1}, \ldots, c_{n} \in \mathbb{R}_{\min }$, where $n$ is equal to the degree of $P, \operatorname{deg} P$. The numbers $c_{1}, \ldots, c_{n}$, are called the corners of $P$. They coincide with the points of nondifferentiability of $\hat{P}$. If $c$ is a corner, the multiplicity of $c$, which is equal to the number of indices $i$ for which $c_{i}=c$, coincides, when $c \neq \mathbb{O}$, with the variation of slope of $\hat{P}$ at $c, \hat{P}^{\prime}\left(c^{-}\right)-\hat{P}^{\prime}\left(c^{+}\right)$. The multiplicity of the corner $\mathbb{O}$ is equal to the valuation of $P$, val $P$. We denote by $\gamma_{1}, \ldots, \gamma_{N}$ the corners of the characteristic polynomial $P_{A}$, that we call the (algebraic) eigenvalues of $A$. Note that the valuation val $P_{A}$ can be computed by introducing the matrix val $A \in \mathbb{R}_{\min }^{n \times n}$, such that (val $\left.A\right)_{i j}=\operatorname{val} A_{i j}$. Then, val $P_{A}$ is equal to the min-plus permanent of the matrix val $A$. By symmetry, the degree $\operatorname{deg} P_{A}$ is equal to the 
max-plus permanent of the matrix $\operatorname{deg} A \in \mathbb{R}_{\max }^{n \times n}$, such that $(\operatorname{deg} A)_{i j}=\operatorname{deg} A_{i j}$. The corners $\gamma_{i}$ (and so, the polynomial function $\hat{P}_{A}$ ) can be computed in $O\left(n^{4} d\right)$ time by adapting the method of Burkard and Butkovic [BB03. (It is not known whether the sequence of coefficients of the formal polynomial $P_{A}$ can be computed in polynomial time.)

For any matrix $B \in \mathbb{R}_{\min }^{n \times n}$ such that perm $B \neq \mathbb{O}$, we define the $\operatorname{graph} \operatorname{Opt}(B)$ as the set of arcs belonging to optimal assignments: the nodes of $\operatorname{Opt}(B)$ are $1, \ldots, n$ and there is an arc from $i$ to $j$ if there is a permutation $\sigma$ such that $j=\sigma(i)$ and $|\sigma|_{B}=\operatorname{perm} B$.

We shall say that two vectors $U, V$ of dimension $n$ with entries in $\mathbb{R}_{\min } \backslash\{\mathbb{O}\}$ form a Hungarian pair with respect to $B$ if, for all $i, j$, we have $B_{i j} \geq U_{i} V_{j}$, and $U_{1} \cdots U_{n} V_{1} \cdots V_{n}=$ perm $B$, the products being understood in the min-plus sense. Thus, $(U, V)$ coincides with the optimal dual variable in the linear programming formulation of the optimal assignment problem (see [BR97, 2.4]). In particular, a Hungarian pair always exists if the optimal assignment problem is feasible, i.e., if perm $B \neq \mathbb{0}$, and it can be computed in $O\left(n^{3}\right)$ time by the Hungarian algorithm. For any Hungarian pair $(U, V)$, we now define the saturation graph, $\operatorname{Sat}(B, U, V)$, which has nodes $1, \ldots, n$ and an arc from $i$ to $j$ if $B_{i j}=U_{i} V_{j}$.

For every finite corner $\gamma$ of $P_{A}$, we define the digraphs $\operatorname{Opt}_{0}(\gamma), \ldots, \mathrm{Opt}_{d}(\gamma)$ : $\operatorname{Opt}_{k}(\gamma)$ has nodes $1, \ldots, n$, and an arc from $i$ to $j$ if $(i, j) \in \operatorname{Opt}(\hat{A}(\gamma))$ and $\left(A_{k}\right)_{i j} \gamma^{k}=\hat{A}_{i j}(\gamma)$. For every Hungarian pair $(U, V)$ with respect to the matrix $\hat{A}(\gamma)$, we also define the digraphs $\operatorname{Sat}_{0}(\gamma, U, V), \ldots, \operatorname{Sat}_{d}(\gamma, U, V)$ by replacing $\operatorname{Opt}(\hat{A}(\gamma))$ by $\operatorname{Sat}(\hat{A}(\gamma), U, V)$, in the definition of $\mathrm{Opt}_{0}(\gamma), \ldots, \mathrm{Opt}_{d}(\gamma)$. Finally, if $G$ is any digraph with nodes $1, \ldots, n$, and if $b \in \mathbb{C}^{n \times n}$, we define the matrix $b^{G}$, which is such that $\left(b^{G}\right)_{i j}=b_{i j}$ if $(i, j) \in G$, and $\left(b^{G}\right)_{i j}=0$ otherwise. In the following theorem, and in the sequel, eigenvalues are counted with multiplicities.

Theorem 1. Let $\gamma$ denote any finite corner of the min-plus characteristic polynomial $P_{A}$. For every $0 \leq k \leq d$, let $G_{k}$ be equal either to $\operatorname{Opt}_{k}(\gamma)$ or $\operatorname{Sat}_{k}(\gamma, U, V)$, for any choice of the Hungarian pair $U, V$ with respect to $\hat{A}(\gamma)$. Consider the auxiliary pencil

$$
a^{(\gamma)}:=a_{0}^{G_{0}}+X a_{1}^{G_{1}}+\cdots+X^{d} a_{d}^{G_{d}} .
$$

Then, if the pencil a ${ }^{(\gamma)}$ has $m_{\gamma}$ non-zero eigenvalues, $\lambda_{1}, \ldots, \lambda_{m_{\gamma}}$, the pencil $\mathcal{A}_{\epsilon}$ has $m_{\gamma}$ eigenvalues $\mathcal{L}_{\epsilon, 1}, \ldots, \mathcal{L}_{\epsilon, m_{\gamma}}$ with respective equivalents of the form $\mathcal{L}_{\epsilon, i} \sim \lambda_{i} \epsilon^{\gamma}$; if 0 is an eigenvalue of multiplicity $m_{\gamma}^{\prime}$ of the pencil $a^{(\gamma)}$, the pencil $\mathcal{A}_{\epsilon}$ has precisely $m_{\gamma}^{\prime}$ eigenvalues $\mathcal{L}_{\epsilon}$ such that $\epsilon^{-\gamma} \mathcal{L}_{\epsilon}$ converges to zero, and all the other eigenvalues $\mathcal{L}_{\epsilon}$ of $\mathcal{A}_{\epsilon}$ are such that the modulus of $\epsilon^{-\gamma} \mathcal{L}_{\epsilon}$ converges to infinity. Moreover, for generic values of the parameters $\left(a_{k}\right)_{i j}, m_{\gamma}$ coincides with the multiplicity of the corner $\gamma$, and $m_{\gamma}^{\prime}$ coincides with the sum of multiplicities of all the corners greater than $\gamma$.

Since the sum of the multiplicities of the corners of $P_{A}$ is equal generically to the degree of $\operatorname{det}\left(\mathcal{A}_{\epsilon}\right)$, and since $\mathcal{A}_{\epsilon}$ has a number of identically zero eigenvalues generically equal to the multiplicity of $\mathbb{O}$ as a corner of $P_{A}$, Theorem 1 provides generically asymptotic equivalents of all the eigenvalues of $\mathcal{A}_{\epsilon}$. 
Example 1. Consider $\mathcal{A}_{\epsilon}=\mathcal{A}_{\epsilon, 0}-X \mathrm{id}$, and

$$
\mathcal{A}_{\epsilon, 0}=\left[\begin{array}{ccc}
b_{11} & b_{12} & b_{13} \\
b_{21} & b_{22} \epsilon & b_{23} \epsilon \\
b_{31} & b_{32} \epsilon & b_{33} \epsilon
\end{array}\right], \text { where } b_{i j} \in \mathbb{C} .
$$

The associated min-plus matrix pencil and characteristic polynomial function are

$$
A=\left[\begin{array}{ccc}
0 \oplus X & 0 & 0 \\
0 & 1 \oplus X & 1 \\
0 & 1 & 1 \oplus X
\end{array}\right], \quad \hat{P}_{A}(x)=(x \oplus 0)^{2}(x \oplus 1)
$$

so that the corners are $\gamma_{1}=\gamma_{2}=0$, with multiplicity 2 , and $\gamma_{3}=1$, with multiplicity 1. We first consider the corner $\gamma=0$. Then $U=V=(0,0,0)$ yields a Hungarian pair with respect to the matrix

$$
\hat{A}(0)=\left[\begin{array}{ccc}
0_{01} & 0_{0} & 0_{0} \\
0_{0} & 0_{1} & 1 \\
0_{0} & 1 & 0_{1}
\end{array}\right]
$$

where we adopt the following convention to visualize the $\operatorname{digraphs}_{\operatorname{Sat}_{k}}(U, V)$ : an arc $(i, j)$ belongs to $\operatorname{Sat}_{k}(U, V)$ if $k$ is put as a subscript of the entry $\hat{A}_{i j}(0)$. For instance, $\hat{A}_{11}(0)=0$, and $(1,1)$ belongs both to $\operatorname{Sat}_{0}(U, V)$ and $\operatorname{Sat}_{1}(U, V)$. Entries without subscripts, like $\hat{A}_{23}(0)=1$, correspond to arcs which do not belong to Sat $(U, V)$. The eigenvalues of the auxiliary pencil $a^{(0)}$ are the roots of

$$
\operatorname{det}\left[\begin{array}{ccc}
b_{11}-\lambda & b_{12} & b_{13} \\
b_{21} & -\lambda & 0 \\
b_{31} & 0 & -\lambda
\end{array}\right]=\lambda\left(-\lambda^{2}+\lambda b_{11}+b_{12} b_{21}+b_{31} b_{31}\right)=0 .
$$

Theorem 1 predicts that this equation has, for generic values of the parameters $b_{i j}$, two non-zero roots, $\lambda_{1}, \lambda_{2}$, which yields two eigenvalues of $\mathcal{A}_{\epsilon}, \mathcal{L}_{\epsilon, m} \sim \lambda_{m} \epsilon^{0}=\lambda_{m}$, for $m=1,2$. Consider finally the corner $\gamma=1$. We can take $U=(0,1,1)$, $V=(-1,0,0)$, and the previous computations become

$$
\hat{A}(1)=\left[\begin{array}{ccc}
0 & 0_{0} & 0_{0} \\
0_{0} & 1_{01} & 1_{0} \\
0_{0} & 1_{0} & 1_{01}
\end{array}\right], \quad \operatorname{det}\left[\begin{array}{ccc}
0 & b_{12} & b_{13} \\
b_{21} & b_{22}-\lambda & b_{23} \\
b_{31} & b_{31} & b_{33}-\lambda
\end{array}\right]=0 .
$$

The latest equation yields $\lambda\left(b_{12} b_{21}+b_{13} b_{31}\right)+b_{12} b_{23} b_{31}+b_{13} b_{32} b_{21}-b_{21} b_{12} b_{33}-$ $b_{31} b_{13} b_{22}=0$. Theorem 10 predicts that this equation has generically a unique nonzero root, $\lambda_{1}$, and that there is a branch $\mathcal{L}_{\epsilon, 1} \sim \lambda_{1} \epsilon$.

As a typical application of Theorem 1 let us consider the following singular perturbation of an affine pencil, $\mathcal{A}_{\epsilon}=\epsilon X^{2} m+X c+k$, already considered in Naj99. For non-zero values of the entries of the matrices $m, c$, and $k$, the associated minplus characteristic polynomial function is $\hat{P}_{A}(x)=(0 \oplus x)^{n}(0 \oplus 1 x)^{n}$. Moreover, the pencils $X c+k$ and $X m+c$ generically both have $n$ finite non-zero eigenvalues, denoted by $\lambda_{1}, \ldots, \lambda_{n}$, and $\mu_{1}, \ldots, \mu_{n}$, respectively. Then, it is easy to derive from Theorem 1 that the pencil $\mathcal{A}_{\epsilon}$ has $n$ eigenvalues $\mathcal{L}_{\epsilon, i} \sim \lambda_{i} \epsilon^{0}$, and $n$ eigenvalues $\mathcal{L}_{\epsilon, i} \sim \mu_{i} \epsilon^{-1}$. Consider now the following non-generic situation. Assume that the pencil $c X+k$ is given, that it is regular, and that its Weierstrass normal form comprises $q_{0}$ Jordan blocks for the eigenvalue 0 , with respective sizes $s_{0}^{1}, \ldots, s_{0}^{q_{0}}$, and $q_{\infty}$ Jordan blocks for the eigenvalue $\infty$, with respective sizes $s_{\infty}^{1}, \ldots, s_{\infty}^{q_{\infty}}$. We set $d_{0}=s_{0}^{1}+\cdots+s_{0}^{q_{0}}, d_{\infty}=s_{\infty}^{1}+\cdots+s_{\infty}^{q_{\infty}}$. We also denote by $q_{0}^{\prime}$ the number of 
one dimensional Jordan blocks for the eigenvalue 0 of the pencil $c X+k$. We denote by $\lambda_{1}, \ldots, \lambda_{r}$ the finite non-zero eigenvalues of $c X+k$ (of course, $r+d_{0}+d_{\infty}=n$ ). We also denote by $\mu_{1}, \ldots, \mu_{t}$ the finite non-zero eigenvalues of the pencil $X m+c$. We say that an eigenvalue $\mathcal{L}_{\epsilon}$ is of order $\epsilon^{\Lambda}$ if $\mathcal{L}_{\epsilon} \sim \lambda \epsilon^{\Lambda}$, for some $\lambda \in \mathbb{C} \backslash\{0\}$. The following result should be compared with Naj99, where partial results are obtained in a similar situation.

Corollary 1. The pencil $\mathcal{A}_{\epsilon}=\epsilon X^{2} m+X c+k$ has precisely

(i) $r$ eigenvalues of order $\epsilon^{0}$, which converge respectively to $\lambda_{i}$, for $i=1, \ldots, r$;

(ii) $t$ eigenvalues or order $\epsilon^{-1}$, which are respectively equivalent to $\mu_{i} \epsilon^{-1}$, for $i=1, \ldots, t$.

It has at least

(iii) $2 q_{0}-q_{0}^{\prime}$ eigenvalues identically equal to zero.

Finally, for generic values of the parameters $m_{i j}$, we have $t=n-q_{\infty}$, and the pencil $\mathcal{A}_{\epsilon}$ has precisely:

(iv) $s_{\infty}^{i}+1$ eigenvalues of order $\epsilon^{-1 /\left(s_{\infty}^{i}+1\right)}$, for $i=1, \ldots, q_{\infty}$;

(v) $s_{0}^{i}-2$ eigenvalues of order $\epsilon^{1 /\left(s_{0}^{i}-2\right)}$, for every $i$ such that $1 \leq i \leq r$ and $s_{0}^{i}>2$.

Corollary 1 provides, for generic values of $m$, the leading exponents of all the eigenvalues of the pencil $\mathcal{A}_{\epsilon}$. In cases $\mathbf{i v} \mathbf{\nabla}$ the generic values of the leading coefficients of the eigenvalues can be determined by formulæ essentially similar to the case of [Lid65, ABG04. This will be detailed elsewhere.

Acknowledgement. The third author thanks Jean-Jacques Loiseau for having suggested to look for a generalization of the result of [ABG04] to matrix pencils.

\section{REFERENCES}

[ABG04] M. Akian, R. Bapat, and S. Gaubert. Generic asymptotics of eigenvalues and minplus algebra. Rapport de recherche 5104, INRIA, Le Chesnay, France, Feb. 2004. Also arXiv:math.SP/0402090

[BB03] R. E. Burkard and P. Butkovič. Finding all essential terms of a characteristic maxpolynomial. Discrete Appl. Math., 130(3):367-380, 2003. Eprint doi:10.1016/S0166-218X(03)00223-3

[BCOQ92] F. Baccelli, G. Cohen, G. Olsder, and J. Quadrat. Synchronization and Linearity an Algebra for Discrete Event Systems. Wiley, 1992.

[BR97] R. Bapat and T. Raghavan. Nonnegative Matrices and Application. Cambridge University Press, 1997.

[CGM80] R. Cuninghame-Green and P. Meijer. An algebra for piecewise-linear minimax problems. Dicrete Appl. Math, 2:267-294, 1980.

[Lid65] V. Lidskiǔ. Perturbation theory of non-conjugate operators. U.S.S.R. Comput. Math. and Math. Phys.,, 1:73-85, 1965. (Z̆. Vyčisl. Mat. i Mat. Fiz. 6, no. 1, 52-60, 1965).

[MBO97] J. Moro, J. V. Burke, and M. L. Overton. On the Lidskii-Vishik-Lyusternik perturbation theory for eigenvalues of matrices with arbitrary Jordan structure. SIAM J. Matrix Anal. Appl., 18(4):793-817, 1997. Eprint doi:10.1137/S0895479895294666

[ME98] Y. Ma and A. Edelman. Nongeneric eigenvalue perturbations of Jordan blocks. Linear Algebra Appl., 273:45-63, 1998. Eprint doi:10.1016/S0024-3795(97)00342-X

[Naj99] B. Najman. The asymptotic behavior of the eigenvalues of a singularly perturbed linear pencil. SIAM J. Matrix Anal. Appl., 20(2):420-427, 1999. Eprint doi:10.1137/S0895479896299949

[VL60] M. I. Višik and L. A. Ljusternik. Solution of some perturbation problems in the case of matrices and self-adjoint or non-selfadjoint differential equations. I. Russian Math. Surveys, 15(3):1-73, 1960. 
Marianne Akian. InRIa, Domaine de Voluceau, B.P. 105, 78153 Le Chesnay Cedex, FRANCE.

E-mail address: Marianne.Akian@inria.fr

Ravindra Bapat. Indian Statistical Institute, New Delhi, 110016, India.

E-mail address: rbb@isid.ac.in

Stéphane Gaubert. InRIA, Domaine de Voluceau, B.P. 105, 78153 Le Chesnay Cedex, France.

E-mail address: Stephane.Gaubert@inria.fr 